\title{
Ultrafast Energy Transfer in an Artificial Photosynthetic Antenna
}

\author{
M. Maiuri ${ }^{1}$, J.J. Snellenburg ${ }^{2}$, I.H.M. van Stokkum ${ }^{2}$, S. Pillai ${ }^{3}$, D. Gust ${ }^{3}$, T.A. Moore ${ }^{3}$, A.L. Moore, \\ R. van Grondelle ${ }^{2}$, G. Cerullo ${ }^{1}$, D. Polli ${ }^{1}$ \\ ${ }^{1}$ IFN-CNR, Dipartimento di Fisica, Politecnico di Milano, Piazza L. da Vinci, 32, 20133 Milano, \\ Italy \\ ${ }^{2}$ Department of Physics and Astronomy, VU University Amsterdam, De Boelelaan 1081, 1081HV \\ Amsterdam, The Netherlands \\ ${ }^{3}$ Department of Chemistry \& Biochemistry and The Center for Bioenergy and Photosynthesis, \\ Arizona State University, Tempe, Arizona 85287-1605, United States
}

\begin{abstract}
We temporally resolved energy transfer kinetics in an artificial lightharvesting dyad composed of a phthalocyanine covalently linked to a carotenoid. Upon carotenoid photo-excitation, energy transfers within $\approx 100 \mathrm{fs}$ ( $\approx 52 \%$ efficiency) to the phthalocyanine.
\end{abstract}

Photosynthesis relies on light harvesting from peripheral antennas -typically performed by carotenoids (Car) and (bacterio)chlorophylls- and subsequent energy transfer (ET) to the reaction center, which can occur with almost $100 \%$ efficiency in some organisms [1]. In this work we studied a prototypical artificial supramolecule (Fig. 1(a)) mimicking the light harvesting process in natural photosynthesis [2]. It is composed of a Car with 10 conjugated double bonds (serving as light harvester) linked to a phthalocyanine ( $\mathrm{Pc}$, acting as energy acceptor) through a phenylamino group [3]. We excite the sample in resonance with the maximum of the first vibronic band of Car and we monitor the ultrafast rise of the Pc population. The $\mathrm{Car} \rightarrow \mathrm{Pc}$ ET process occurs from the bright Car $\mathrm{S}_{2}$ excited state and competes with an internal conversion (IC) process towards the lower-lying dark Car $\mathrm{S}_{1}$ excited state. To establish the relative weight of these two deactivation pathways, we compare the excited state dynamics of the isolated Car (where only the IC process occurs) with that of the dyad (where also the ET channel is active). Both these processes occur with sub-100-fs time constants, thus challenging the time resolution of conventional transient absorption (TA) systems. We overcome this limit by exploiting a state-of-the-art pump-probe system with 10-fs temporal resolution. We were able to extract a $\approx 52 \% \mathrm{ET}$ efficiency, one of the highest values so far recorded for energy transfer from the $\mathrm{S}_{2}$ state in artificial complexes.

Our experimental apparatus is based on two synchronized non-collinear optical parametric amplifiers (NOPAs). The first NOPA generates 10-fs pulses in the green, resonant with the $\mathrm{S}_{0} \rightarrow \mathrm{S}_{2}$ transition of the Car, while the second NOPA provides ultra-broadband probe pulses with $\approx 7-\mathrm{fs}$ duration spanning the 500-700 $\mathrm{nm}$ wavelength range, thus covering both the Car and Pc absorption bands [4]. A spectrometer with single-shot detection capability at $1 \mathrm{kHz}$ is used to acquire 2dimensional TA maps as a function of probe wavelength and delay [5].

This is an Open Access article distributed under the terms of the Creative Commons Attribution License 2.0, which permits unrestricted use, distribution, and reproduction in any medium, provided the original work is properly cited. 

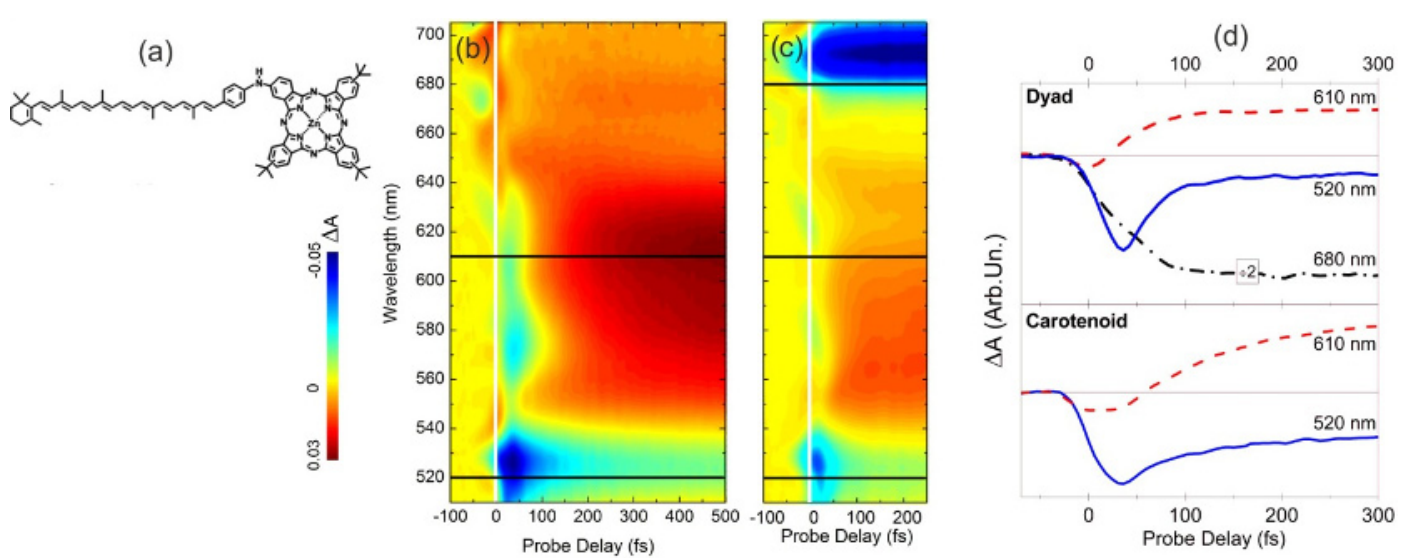

Fig. 1. (a) molecular structure of the dyad; (b) and (c) $\Delta \mathrm{A}$ maps as a function of probe wavelength and delay for the isolated carotenoid and the dyad respectively; (d) temporal time traces at selected probe wavelengths for the isolated carotenoid (bottom panel) and dyad (upper panel).

Fig. 1(b) shows the $\Delta \mathrm{A}$ map of the isolated Car in solution: we observe the prompt rise of a negative signal, which we assign to ground state photobleaching (PB) and stimulated emission (SE) from $S_{2}$ to $S_{0}$. This signal rapidly decays, giving rise to the formation of a positive photoinduced absorption (PA) band peaking at $610 \mathrm{~nm}$, which is completed within $\approx 400 \mathrm{fs}$. This PA band is well known in Cars and assigned to a transition from $S_{1}$ to an higher-lying $S_{n}$ state, thus providing a signature of the population in the $S_{1}$ state through the IC process. The PA band shows a faster buildup in the red compared to the blue, and undergoes spectral narrowing within the first $500 \mathrm{fs}$. These effects are due to intermolecular vibrational relaxation within $S_{1}$ associated with dissipation of excess energy deposited as a result of the IC process.

In the dyad (Fig. 1(b)) the temporal evolution of the TA signals occurs on a significantly shorter time scale as compared to the isolated Car, indicating a much faster decay of the population from the initially excited Car $\mathrm{S}_{2}$ state. In particular, the resulting TA spectrum, completed within $\approx 100 \mathrm{fs}$, displays not only the expected $\mathrm{S}_{1} \rightarrow \mathrm{S}_{\mathrm{n}}$ PA from the Car but also: (i) a peaked negative PB signal around $690 \mathrm{~nm}$, reflecting the Pc ground state absorption spectrum and thus indicating Car $\rightarrow \mathrm{Pc}$ ET; (ii) an increased PA shoulder in the blue (peaking at $\approx 555 \mathrm{~nm}$ ), representing a new deactivation pathway within the Car manifold towards an additional intermediate excited state denoted $\mathrm{S}^{*}$. This state, which was not participating in the IC process for the isolated Car, had already been observed for other Cars when embedded in light-harvesting systems in the same spectral region [6].
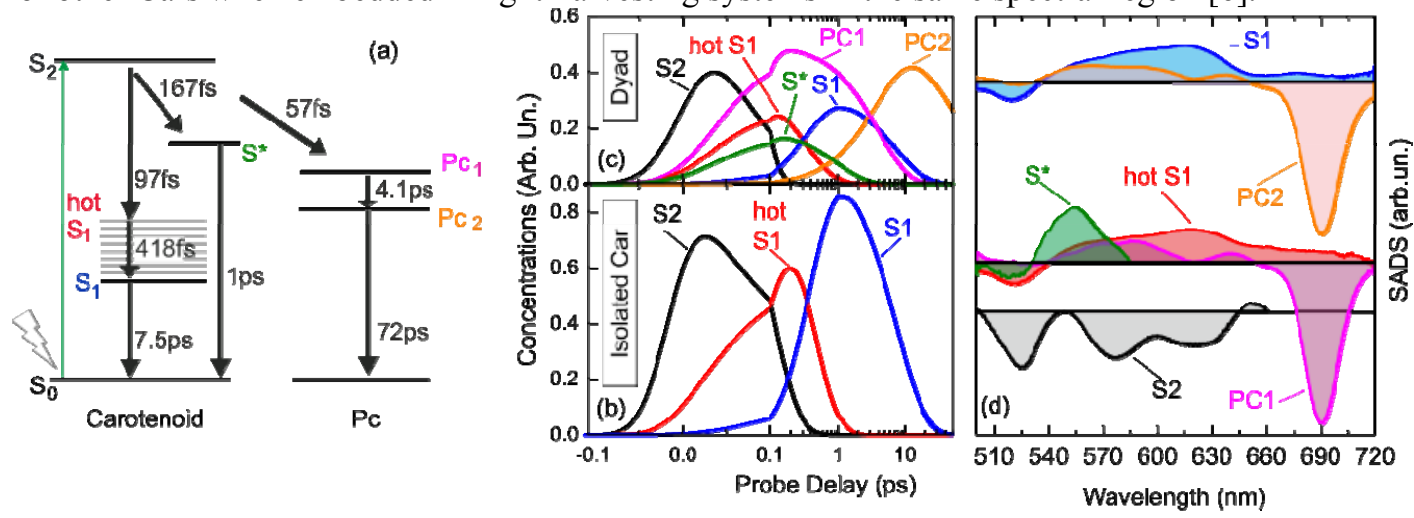

Fig. 2. (a) Energy level diagram in the target analysis; the numbers on the thick arrows indicate the inverse of the rate constants of the deactivation pathways involved; (b-c) Temporal evolution of the concentration profiles for the isolated Car (b) and for the dyad (c); the time axis is linear until $0.1 \mathrm{ps,} \mathrm{then} \mathrm{logarithmic;} \mathrm{(d)} \mathrm{Estimated}$

SADS. 
The two datasets for Car and the dyad were analyzed simultaneously using a combination of global and target analysis [7]. The compartmental model used is depicted in Fig. 2(a). $\mathrm{S}_{1}{ }^{\text {hot }} \rightarrow \mathrm{S}_{1}$ and $\mathrm{Pc}_{1} \rightarrow \mathrm{Pc}_{2}$ spectral evolutions were taken into account in order to include vibrational relaxation processes within the two species. $S_{2} \rightarrow S_{1}{ }^{\text {hot }} \rightarrow S_{1}$ IC rate constants and associated SADS were constrained to be equal for the isolated Car and the dyad. The result is a combination of concentration profiles (Fig. 2 (b-c)) and their respective spectral amplitudes. In Fig. 2 (d) these estimated species associated difference spectra (SADS) are shown. The first SADS can be identified as the $S_{2}$ of the Car with a PB at $525 \mathrm{~nm}$ and SE from 550 until $650 \mathrm{~nm}$. From the $\mathrm{S}_{2}$ state of the Car three different species are populated: the hot $\mathrm{S}_{1}$ of the Car, the first state of the Pc species, and a clear Car $\mathrm{S}^{*}$ state. The $\mathrm{S}^{*}$ state is populated by the $\mathrm{S}_{2}$ state and subsequently decays again in about 1 ps to the ground state, presumably via an IC processes. The Car S* state is restricted to only contribute to the fit below $580 \mathrm{~nm}$. The hot $\mathrm{S}_{1}$ state evolves in roughly 400 fs into the $S_{1}$ state which subsequently decays to the ground state in 7.5 ps. The resulting ET efficiency from the Car $\mathrm{S}_{2}$ state to the Pc molecules is then calculated as $\eta_{E T}=\frac{1 / 57}{1 / 57^{+1 / 97^{+1 / 167}}}=51.8 \%$.

In conclusion, using our state-of-the-art spectroscopy system with $\approx 10$-fs temporal resolution it was possible to completely time resolve the ultrafast excited-state dynamics of the isolated Car and of the dyad system, occurring on the $\approx 100$-fs time scale. Their simultaneous comparison by means of global and target analysis enabled the evaluation of ET pathways and efficiency (as high as $\approx 50 \%$ ) from the Car to the Pc.

\section{References}

[1] H.A. Frank, R.J. Cogdell, Photochem. Photobiol. 63, 257 (1996)

[2] D. Gust, T.A. Moore, A.L. Moore, C. Devadoss, P.A. Liddell, R. Hermant, R.A. Nieman, L.J. Demanche, J.M. Degraziano, I. Gouni, J. Am. Chem. Soc. 114, 3590 (1992)

[3] M. Kloz, S. Pillai, G. Kodis, D. Gust, T.A. Moore, A.L. Moore, R. van Grondelle, J.T. Kennis, J. Am. Chem. Soc. 133, 7007 (2011)

[4] C. Manzoni, D. Polli, G. Cerullo, Rev. Sci. Instrum. 77, 023103 (2006)

[5] D. Polli, L. Lüer, G. Cerullo, Rev. Sci. Instrum., 78, 103108 (2007)

[6] C.C. Gradinaru, J.T.M. Kennis, E. Papagiannakis, I.H.M. van Stokkum, R.J. Cogdell, G.R. Fleming, R.A. Niederman, R. van Grondelle, Proc. Natl. Acad. Sci. USA 98, 2364 (2001)

[7] I.H.M. van Stokkum, D.S. Larsen, R. van Grondelle, Biochim. Biophys. Acta 1657, 82 (2004) 